a short bibliography. The paucity of up to date literature indicates the dearth of current research (in fact geriatrics is one of the fields 'wide open' for research).

The book is suitable for students and those doing junior geriatric appointments whether as potential geriatric physicians or 'en passant' for some other branch of medicine.

\section{Diagnosis and Treatment of Cardiac Arrhythmias}

By J. P. P. Stock. Second edition. Pp. 271, illustrated. London: Butterworths, 1970. £3.90.

With the increasing range of effective methods of treatment, cardiac arrhythmias provide the clinician with frequent opportunities for doing his patient good-or harm. In practice the causes of individual arrhythmias are frequently complex and therapy is all too often a matter of trial and error. However, enough is now known about the mechanisms of arrhythmias and therapy to permit a rational approach to management that will shorten the length of trial and minimize the likelihood of error.

Dr Stock's book, first published in 1969, was soon recognized as an outstanding contribution to the literature in this field. The second edition is enlarged with a new chapter on the Wolff-Parkinson-White syndrome and its associated arrhythmias. The book is a happy marriage of accounts of the anatomy and electrophysiology of rhythm disturbances, and of the practical aspects of recognition and management. There is an extensive and remarkably up to date bibliography which is a tribute to both author and publisher.

The text is eminently readable with a continuity and momentum that makes the book difficult to put down. With two editions in successive years, Dr Stock's book is fast turning into a classic and deservedly so.

\section{Venography of the Inferior Vena Cava and its Branches}

By Ernest J. Ferris, florencio A. Hipona, Paul C. Kahn, Ervin Philipps and Jerome H. Shapiro. Pp. 229, illustrated. Baltimore: Williams \& Wilkins. Edinburgh \& London: E. \& S. Livingstone, 1970. £7.50.

This book is an invaluable record of the experiences of its authors of the investigations and radiological appearances of the inferior vena cava. It is essentially a reference book for the surgeon and radiologist who have a particular interest in this field of investigation, but it is written in such a manner that it would prove worthwhile and enjoyable reading for anyone whose interests lie on the fringe of this subject.

Each of the five authors describes, in detail, one aspect of the radiology of the inferior vena cava. In the first chapter, the embryology is clearly described. Information is conveye largely in the form of diagrams, which, although wet presented, have the disadvantage of employing initials an $\$$ abbreviations necessitating constant reference to the key? This is followed by a useful description of the normal. anatomy and its variations.

In the chapter on techniques, there is very little mentiof of the intra-osseous approach, which, in my experience्e plays an important part in the visualization of the inferiod vena cava and its tributaries, when the femoral vein occluded. Although this method is referred to in the tex practical details are not given.

The diagrams of collateral circulation are excellent although with the same disadvantage of the extensive use of initials.

Overall, this book is a first-rate collection of examples of diseases and abnormalities affecting the inferior vena cavat Of its 229 pages, 115 are largely occupied by radiographs These are of a high quality and are clearly presented ang described. In addition to their obvious value, when faced with an unusual radiological appearance in the venous system, they are an important reminder of the diverse diseases which can be investigated in this way.

A disproportionately large amount of space is devoted to the appearances (of the vena cava), after ligation and plicat tion, but it is regrettable that no mention is made of thd techniques and appearances of operative venography whic $\delta$ are so important during the exploration of the diseased vein No doubt this can be amended in the second edition.

\section{Principles of Chest X-Ray Diagnosis}

By George Simon. Third edition. Pp. 271, illustraced London: Butterworths, 1971. $£ 8.00$.

The third edition of this well known classic book exced the standard of its predecessors and must remain prescribe reading for all radiologists and chest physicians in training New material includes helpful line diagrams and sections om segmental lesions, lung infarcts, emphysema and plain filp appearances in heart disease. Those reared on the firse edition, like the reviewer, may be surprised to find how valuable these are.

From the manner of its presentation, considered from the radiological appearances, it is invaluable as a reference book (though it quite properly has never set out to be an atlas, for all clinicians, and this new edition should be available all hospital and medical libraries. 